\title{
Mapping EELS Vibrational Modes in MgO Nanocubes
}

M. J. Lagos ${ }^{1,2,3}$, P. E. Batson ${ }^{1,2,3}$

${ }^{1 .}$ Inst. for Advanced Materials, Devices and Nanotechnology, Rutgers University, Piscataway, NJ, USA.

2. Department of Physics and Astronomy, Rutgers University, Piscataway, NJ, USA.

${ }^{3 .}$ Department of Materials Science and Engineering, Rutgers University, Piscataway, NJ, USA.

Both experimental and theoretical studies of vibrational modes in ionic crystals have been intensively conducted over the last five decades. Theoretical approaches, using lattice dynamic and classical electrodynamics, determined the vibrational normal modes and predicted macroscopic and microscopic vibrational modes of crystal bulk/surfaces. For instance, Fuchs and Kliewer predicted the existence of a surface mode, whose oscillation frequency lies between the regular transverse (TO) and longitudinal optical (LO) modes [1]. Experimentalists found these surface vibrational modes in several materials, as a result of the coupling between the external excitation probe (photons, electrons, etc.) and the solid phonon modes. In fact, significant progress in the understanding of the physics of vibrational phonon modes of solids has been achieved in the last fifty years. In spite of these significant advances, our knowledge of the spatial extent of optical vibrational modes in nano-structures is still being built and the nano-phononic field remains to be further explored. In this work, we present a study of the spatial distribution of EELS excitations of vibrational mode in $\mathrm{MgO}$ nanocubes.

We have used a Nion UltraStem equipped with a monochromator to study the spatial dependence of vibrational modes in nanostructures using a $\sim 2 \AA$ probe with energy spread of $\sim 10 \mathrm{meV}$, at $60 \mathrm{KV}$ [2]. In particular, we have studied the vibrational response of nanometric-size magnesium oxide cubes, as a function of electron beam position. These spatially-resolved results provide data to build 2-D maps of energy loss scattering to vibrational modes.

Our spatially-resolved experimental results indicate that $\mathrm{MgO}$ nanocubes support a variety of vibrational modes: (i) It can sustain both transverse and transverse optical excitation modes, similar to those observed in bulk $\mathrm{MgO}$ [3]. Those modes have excitation energies around 50 and $90 \mathrm{meV}$, respectively, and involve the collective oscillation of the oxygen sub-lattice against the magnesium sublattice. These vibrational excitations are mainly located in the inner regions of the nanocube. (ii) It can also support surface macroscopic Fuchs-Kliewer $(\sim 80 \mathrm{meV})$ and Edge $(\sim 70 \mathrm{meV})$ modes, which are localized next to the surface. Our results point out that inside the cube the decay length of those excitations are smaller than about $15 \mathrm{~nm}$. Interestingly, those surface modes can also be excited in the aloof geometry, as shown in figure 1. All the aforementioned energy loss excitations can be interpreted in terms of the eigenmodes of cubic nanostructure, within the dielectric formulation [4].

Our results provide progress in understanding the spatial variation of vibrational modes of nanostructures as a result of swift electron-phonon coupling, pointing out the possibility of wide exploration of highly localized vibrational excitations in nano-phononic crystals. [5]

References:

[1] R.Fuchs and K.L.Kliewer, Physical Review 140 (1965), p. A2076.

[2] O. Krivanek, et al, Nature 514 (2014), p. 209.

[3] G.Peckham, Proc. Phys. Soc. 90 (1967), p. 657. 
[4] H. Ibach, D. L. Mills, EELS and Surface Vibrations, Academic Press (1982).

[5] We gratefully acknowledge extensive advice and help with the new Nion equipment by T.C. Lovejoy, N. Dellby, and O.L. Krivanek. This research was supported by the U.S. Department of Energy, Office of Science, Basic Energy Sciences under Award \# DE-SC0005132.

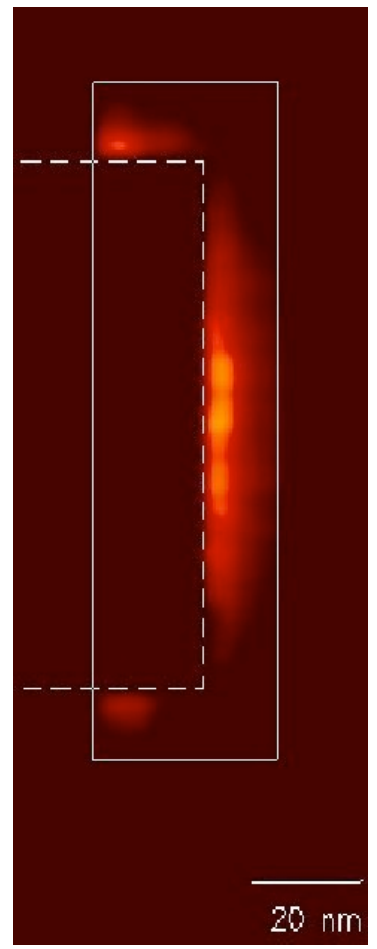

(a)

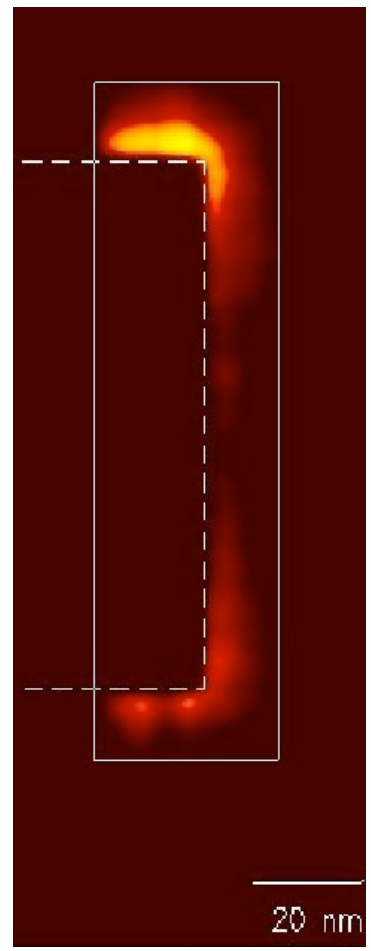

(b)

Figure 1. EELS scattering maps associated with surface vibrational excitations in a $100 \mathrm{~nm} \mathrm{MgO}$ cube. Surface Fuchs-Kliewer (a) and Edge (b) modes are excited at about $80 \mathrm{meV}$ and $70 \mathrm{meV}$, respectively. The cube is indicated by the dotted line and the map was built using only excitations induced by the electron beam in the aloof geometry acquired in the rectangular region. Note that the strength decays in the outward direction, probably due to the evanescent characteristics of the oscillating electrostatic field associated with the surface vibrational mode. 